\title{
Motivación percibida por los adolescentes gallegos en la práctica del ejercicio
}

\section{físico}

\section{Perceived motivation by galician adolescents in the practice of physical exercise}

\author{
Iago Portela Pino*, José Domínguez Alonso* \\ *Universidade de Vigo
}

\begin{abstract}
Resumen
El presente trabajo tiene como objetivo identificar las motivaciones que llevan a los adolescentes a incrementar la práctica del ejercicio físico. Para ello, se aplicó el cuestionario de Autionforme de Motivos para la Práctica de Ejercicio Físico (AMPEF) a 342 estudiantes de Educación Secundaria Obligatoria y Bachillerato $(\mathrm{M}=15.02 \pm 1.86)$. Los resultados evidencian que prevención/salud positiva y diversión/bienestar, son los principales factores motivacionales en la práctica del ejercicio físico. Finalmente, con menor impacto en la motivación se encuentra el reconocimiento social/desafío y urgencias de salud. En conclusión, conocer los motivos que apoyan la práctica regular del ejercicio físico puede ayudar a reducir el sedentarismo.
\end{abstract}

Palabras clave: motivación, ejercicio físico, adolescentes, AMPEF.

\begin{abstract}
This paper aims to identify the motivations that lead adolescents to increase the practice of physical exercise. To do this, applied the questionnaire of Autionforme's reasons for the practice of physical exercise (AMPEF) 342 students of compulsory secondary education $(\mathrm{M}=15.02 \pm 1.86)$. The results show that positive prevention/health and fun/welfare, are the main motivational factors in the practice of physical exercise. Finally, with less impact on motivation social recognition is / challenge and health emergency. In conclusion, ascertain the reasons which support the regular practice of physical exercise can help to reduce physical inactivity.

Keywords: motivation, physical exercise, adolescents, AMPEF.
\end{abstract}

La motivación parece ser la pieza clave en la elección de determinadas actividades y en las causas que determinan la permanencia o abandono de las mismas. Las etapas de la Educación Secundaria Obligatoria (ESO) y el Bachillerato son un período muy apropiado para la labor de promoción del ejercicio físico, pues en ellas se produce un gran porcentaje de abandono de la práctica deportiva entre el alumnado. De hecho, identificar los factores motivacionales para la práctica del ejercicio físico se ha convertido en un importante campo de investigación en los últimos años (Cecchini, 2011; Jaakkola, Washington \& Yli-Piipari, 2013; Martínez et al., 2012).

En consecuencia, entre los factores que pueden elevar la motivación hacia la práctica del ejercicio físico en edades adolescentes se encuentran: la presión de grupo, algunos videojuegos, el acceso fácil a un gimnasio, dirección inicial por un entrenador experto, la variedad de actividades, deportes en equipo, participación de los apoyos familiares y entre compañeros (Peeters et al., 2012), o la sensación de satisfacción después de la pérdida de peso (Vergara et al., 2012). De hecho, los factores motivadores más importantes parecen ser el grupo de amigos, el entrenador y la escuela (Kulavic, Hultquist \& McLester, 2013).

Así pues, el objetivo del presente estudio es conocer los factores motivacionales que contribuyen a optimizar el inicio y la permanencia de la práctica del ejercicio físico en adolescentes, así como disminuir su abandono.

\section{Método}

\section{Participantes}

La población total objeto de este estudio compuesta por 342 adolescentes de la Comunidad Autónoma de Galicia, distribuyéndose en función del género en 157 chicas (45.9\%) y 185 chicos $(54.1 \%)$, con edades comprendidas entre 12 y 18 años (26.9\% tiene 12 o 13 años, $24.6 \%$ tiene 14 o 15 años, y un $48.5 \%$ tiene 16,17 o 18 años), siendo la media de edad 15.1 años $(\mathrm{DT}=1.86)$.

\section{Instrumento}

El instrumento utilizado fue el cuestionario Autionforme de Motivos para la Práctica de Ejercicio Físico (AMPEF), escala adaptada al español por Capdevila, Niñerola y Pintanel (2004) a partir del Exercise Motivations Inventory-2 (EMI-2) de Markland e Ingledew (1997). Consta de cuarenta y ocho ítems agrupados en once factores: peso e imagen corporal, diversión y bienestar, prevención y salud positiva, competición, afiliación, fuerza y resistencia muscular, reconocimiento social, control del estrés, agilidad y flexibilidad, desafío, y urgencias de salud. Su formato de respuesta es tipo Likert de cero (nada verdadero) a diez (totalmente verdadero). El cuestionario considerado en su totalidad para la muestra, presenta un índice de fiabilidad muy alto $(\alpha=.96)$.

\section{Procedimiento}

El cuestionario se administró de manera colectiva en estudiantes de educación secundaria obligatoria y bachillerato en horario regular de clases, durante el curso 
2015-16. Después de comunicar las instrucciones oportunas y previo consentimiento informado (centro y familias), todo el alumnado cumplimentó de forma voluntaria la información solicitada. Se cumplieron los protocolos éticos de investigación con especial énfasis en la confidencialidad.

\section{Análisis de los datos}

Para el análisis de los datos se emplearon el programa estadístico SPSS 21.0 (IBM Corp., 2012). Se llevó a cabo un análisis descriptivo de los ítems, media, desviación típica, así como de los porcentajes obtenidos en cada afirmación.

\section{Resultados}

En el AMPEF, las puntuaciones parciales de cada ítem ofrecen información sobre el tipo de motivación o predisposición que favorece en una persona la práctica del ejercicio físico (Tablas 1 y 2). Así pues, las puntuaciones más elevadas se presentan en los ítems "porque me hace sentir bien" ( $M=4.97$; Totalmente verdadero: 28.9\%), "para mantenerme sano" ( $\mathrm{M}=4.90$; Totalmente verdadero: $24.6 \%)$, "para tener un cuerpo sano" ( $\mathrm{M}=4.87$; Totalmente verdadero: $23.7 \%)$ y "para sentirme más sano" $(\mathrm{M}=4.74$; Totalmente verdadero: $27.8 \%$ ), todos ellos forman parte del factor prevención/salud positiva, siendo considerado el motivo principal que lleva a la práctica del ejercicio físico en los adolescentes. A continuación, los ítems "porque me gusta la sensación que tengo al hacer ejercicio" (M=4.73; Totalmente verdadero: $26 \%)$, "porque el ejercicio me produce diversión” ( $M=4.70$; Totalmente verdadero: $26.9 \%$ ) y "porque haciendo ejercicio me siento muy bien" ( $\mathrm{M}=4.68$; Totalmente verdadero: $29.2 \%)$, que forman parte del factor afiliación/diversión/bienestar, considerado otro de los factores determinantes en la práctica del ejercicio físico en esta población.

Tabla 1.

Descriptivos de los items del AMPEF con el total de la muestra de ESO y Bachiller $(N=342)$, por orden de frecuencia (Puestos $1^{\circ}$ a $24^{\circ}$ )

\begin{tabular}{|c|c|c|c|c|c|c|c|c|c|c|c|c|}
\hline \multirow{2}{*}{ Orden } & \multirow{2}{*}{ Ítem } & \multirow{2}{*}{ Factor } & \multirow{2}{*}{ Enunciado } & \multirow{2}{*}{$\mathrm{M}$} & \multirow{2}{*}{ D.T. } & \multicolumn{7}{|c|}{ Escala (\%) } \\
\hline & & & & & & 1 & 2 & 3 & 4 & 5 & 6 & 7 \\
\hline $1^{\circ}$ & 3 & $\mathrm{P} / \mathrm{SP}$ & Porque me hace sentir bien & 4.97 & 1.88 & 8.8 & 3.2 & 9.6 & 14 & 18.1 & 17.3 & 28.9 \\
\hline $2^{\circ}$ & 2 & $\mathrm{P} / \mathrm{SP}$ & Para mantenerme sano & 4.90 & 1.76 & 7 & 3.2 & 10.2 & 16.4 & 23.1 & 15.5 & 24.6 \\
\hline $3^{\circ}$ & 5 & $\mathrm{P} / \mathrm{SP}$ & Para tener un cuerpo sano & 4.87 & 1.75 & 5.6 & 5.3 & 12 & 14.3 & 23.4 & 15.8 & 23.7 \\
\hline $4^{\circ}$ & 32 & $\mathrm{P} / \mathrm{SP}$ & Para sentirme más sano & 4.74 & 2.06 & 12.6 & 6.1 & 9.4 & 10.5 & 17.5 & 16.1 & 27.8 \\
\hline $5^{\circ}$ & 7 & $\mathrm{~A} / \mathrm{D} / \mathrm{B}$ & $\begin{array}{l}\text { Porque me gusta la sensación que } \\
\text { tengo al hacer ejercicio }\end{array}$ & 4.73 & 2.05 & 13.2 & 4.7 & 9.9 & 11.1 & 16.1 & 19 & 26 \\
\hline $6^{\circ}$ & 34 & $\mathrm{~A} / \mathrm{D} / \mathrm{B}$ & $\begin{array}{l}\text { Porque el ejercicio me produce } \\
\text { diversión }\end{array}$ & 4.70 & 2.01 & 10.8 & 7 & 8.5 & 17.3 & 14.3 & 15.2 & 26.9 \\
\hline $7^{\circ}$ & 45 & $\mathrm{~A} / \mathrm{D} / \mathrm{B}$ & $\begin{array}{l}\text { Porque haciendo ejercicio me } \\
\text { siento muy bien }\end{array}$ & 4.68 & 2.09 & 13.2 & 5.6 & 11.1 & 13.2 & 13.7 & 14 & 29.2 \\
\hline $8^{\circ}$ & 20 & $\mathrm{~F} / \mathrm{RM}$ & Para aumentar mi resistencia & 4.50 & 2.03 & 14.3 & 6.4 & 9.1 & 14.3 & 17.8 & 17.3 & 20.8 \\
\hline $9^{\circ}$ & 19 & $\mathrm{P} / \mathrm{SP}$ & $\begin{array}{l}\text { Porque quiero disfrutar de buena } \\
\text { salud }\end{array}$ & 4.46 & 1.97 & 13.2 & 5 & 11.4 & 18.7 & 17.3 & 13.7 & 20.8 \\
\hline $10^{\circ}$ & 11 & $\mathrm{~A} / \mathrm{F}$ & Para estar más ágil & 4.39 & 1.91 & 12.9 & 5.3 & 12 & 19.3 & 17.5 & 16.4 & 16.7 \\
\hline $11^{\circ}$ & 35 & $\mathrm{~A} / \mathrm{D} / \mathrm{B}$ & $\begin{array}{l}\text { Para divertirme haciendo } \\
\text { ejercicio con otras personas }\end{array}$ & 4.34 & 2.13 & 18.4 & 5.8 & 8.2 & 16.1 & 15.2 & 14.6 & 21.6 \\
\hline $12^{\circ}$ & 6 & $\mathrm{~F} / \mathrm{RM}$ & Para tener más fuerza & 4.33 & 2.15 & 17 & 8.5 & 9.1 & 15.2 & 14.3 & 12.3 & 23.7 \\
\hline $13^{\circ}$ & 21 & $\mathrm{P} / \mathrm{SP}$ & $\begin{array}{l}\text { Porque el ejercicio hace que me } \\
\text { sienta satisfecho }\end{array}$ & 4.31 & 2.07 & 15.5 & 6.7 & 14 & 14 & 15.5 & 13.5 & 20.8 \\
\hline $14^{\mathrm{o}}$ & 14 & $\mathrm{P} / \mathrm{SP}$ & Para evitar problemas de salud & 4.26 & 2.10 & 17.8 & 7 & 10.8 & 12.9 & 19 & 12.3 & 20.2 \\
\hline $15^{\circ}$ & 16 & $\mathrm{P} / \mathrm{IC}$ & Para tener buen cuerpo & 4.25 & 2.15 & 18.7 & 5.8 & 14 & 11.7 & 13.5 & 15.5 & 20.8 \\
\hline $16^{\mathrm{o}}$ & 48 & $\mathrm{C} / \mathrm{RS} / \mathrm{D}$ & Para probarme a mi mismo & 4.20 & 2.18 & 19.9 & 7.6 & 10.5 & 12.6 & 15.5 & 12.3 & 21.6 \\
\hline $17^{\circ}$ & 12 & $\mathrm{C} / \mathrm{RS} / \mathrm{D}$ & $\begin{array}{l}\text { Para tener unas metas por las que } \\
\text { esforzarme }\end{array}$ & 4.14 & 2.11 & 18.1 & 8.2 & 13.7 & 12.3 & 15.5 & 13.2 & 19 \\
\hline $18^{\circ}$ & 8 & $\mathrm{~A} / \mathrm{D} / \mathrm{B}$ & $\begin{array}{l}\text { Para pasar el tiempo con los } \\
\text { amigos }\end{array}$ & 4.12 & 2.12 & 20.2 & 5.6 & 12.9 & 15.2 & 14.6 & 12.9 & 18.7 \\
\hline $19^{\circ}$ & 15 & $\mathrm{P} / \mathrm{SP}$ & Porque el ejercicio me da energía & 4.11 & 2.11 & 19.6 & 7.6 & 11.4 & 14 & 15.5 & 14.3 & 17.5 \\
\hline $20^{\circ}$ & 40 & $\mathrm{P} / \mathrm{IC}$ & Para quemar calorías & 4.07 & 2.19 & 21.1 & 9.1 & 9.9 & 14.6 & 13.7 & 10.5 & 21.1 \\
\hline $21^{\mathrm{o}}$ & 33 & $\mathrm{~F} / \mathrm{RM}$ & Para ser más fuerte & 4.02 & 2.14 & 20.2 & 8.5 & 12.6 & 16.4 & 12.9 & 9.4 & 20.2 \\
\hline $22^{\circ}$ & 43 & $\mathrm{CE}$ & Para liberar la tensión & 4.01 & 2.23 & 23.7 & 7.9 & 9.9 & 13.7 & 13.5 & 9.9 & 21.3 \\
\hline $23^{\circ}$ & 39 & $\mathrm{C} / \mathrm{RS} / \mathrm{D}$ & $\begin{array}{l}\text { Para desarrollar mis habilidades } \\
\text { personales }\end{array}$ & 3.94 & 2.09 & 19.9 & 10.8 & 10.5 & 15.5 & 16.4 & 10.8 & 16.1 \\
\hline $24^{\circ}$ & 26 & $\mathrm{C} / \mathrm{RS} / \mathrm{D}$ & Para tener retos que superar & 3.89 & 2.22 & 24.9 & 7.6 & 12 & 12.9 & 12.6 & 10.5 & 19.3 \\
\hline
\end{tabular}

P/SP: Prevención/Salud positiva; C/RS/D: Competición/Reconocimiento social/Desafío; P/IC: Peso/Imagen corporal; A/D/B:

Afiliación/Diversión/Bienestar; CE: Control del estrés; F/RM: Fuerza/Resistencia muscular; US: Urgencias de salud; A/F:

Agilidad/Flexibilidad.

Interpretación escala: 1. Nada, 2. Poco, 3. Algo, 4. Regular, 5. Bastante, 6. Casi totalmente, 7. Totalmente 
Del mismo modo, en un nivel intermedio de puntuación se encuentran motivos muy dispersos como "para desarrollar mis habilidades personales" (M=3.94; Totalmente verdadero: $16.1 \%$ ) o "para tener retos que superar" ( $\mathrm{M}=3.89$; Totalmente verdadero: $19.3 \%)$ del factor competición/reconocimiento social/desafio; "para ayudarme a superar el estrés" ( $\mathrm{M}=3.89$; Totalmente verdadero: 19.3\%) del factor control del estrés; y "para desarrollar mis músculos" $(\mathrm{M}=3.88$; Totalmente verdadero: $18.4 \%$ ) del factor fuerza/resistencia muscular; y "para mejorar mi aspecto" (M=3.83; Totalmente verdadero: $17.3 \%$ ) del factor peso/imagen corporal.

Tabla 2.

Descriptivos de los items del AMPEF con el total de la muestra de ESO y Bachiller $(N=342)$, por orden de frecuencia (Puestos $24^{\circ}$ a $48^{\circ}$ )

\begin{tabular}{|c|c|c|c|c|c|c|c|c|c|c|c|c|}
\hline \multirow{2}{*}{ Orden } & \multirow{2}{*}{ Ítem } & \multirow{2}{*}{ Factor } & \multirow{2}{*}{ Enunciado } & \multirow{2}{*}{ M } & \multirow{2}{*}{ D.T. } & \multicolumn{7}{|c|}{ Escala (\%) } \\
\hline & & & & & & 1 & 2 & 3 & 4 & 5 & 6 & 7 \\
\hline $25^{\circ}$ & 31 & $\mathrm{CE}$ & Para ayudarme a superar el estrés & 3.89 & 2.23 & 24.9 & 9.1 & 9.9 & 13.2 & 13.5 & 10.2 & 19.3 \\
\hline $26^{\circ}$ & 44 & $\mathrm{~F} / \mathrm{RM}$ & Para desarrollar mis músculos & 3.88 & 2.17 & 22.8 & 9.1 & 14 & 11.7 & 14.6 & 9.4 & 18.4 \\
\hline $27^{\circ}$ & 29 & $\mathrm{P} / \mathrm{IC}$ & Para mejorar mi aspecto & 3.83 & 2.17 & 24.6 & 8.8 & 11.1 & 14.9 & 13.5 & 9.9 & 17.3 \\
\hline $28^{\circ}$ & 37 & $\mathrm{C} / \mathrm{RS} / \mathrm{D}$ & $\begin{array}{l}\text { Porque disfruto haciendo } \\
\text { competición física }\end{array}$ & 3.80 & 2.14 & 22.8 & 10.8 & 11.7 & 14.9 & 14.3 & 8.5 & 17 \\
\hline $29^{\circ}$ & 24 & $\mathrm{C} / \mathrm{RS} / \mathrm{D}$ & $\begin{array}{l}\text { Porque me lo paso bien } \\
\text { compitiendo }\end{array}$ & 3.78 & 2.21 & 26.9 & 6.4 & 14 & 12.3 & 12.6 & 9.9 & 17.8 \\
\hline $30^{\circ}$ & 27 & $\mathrm{P} / \mathrm{IC}$ & Para controlar mi peso & 3.75 & 2.22 & 27.2 & 7.3 & 14.3 & 10.2 & 14 & 8.8 & 18.1 \\
\hline $31^{\circ}$ & 47 & $\mathrm{C} / \mathrm{RS} / \mathrm{D}$ & $\begin{array}{l}\text { Porque me divierte hacer } \\
\text { ejercicio sobre todo si hay } \\
\text { competición }\end{array}$ & 3.73 & 2.32 & 29.8 & 9.4 & 9.4 & 9.9 & 11.7 & 10.2 & 19.6 \\
\hline $32^{\circ}$ & 1 & $\mathrm{P} / \mathrm{IC}$ & Para mantenerme delgado & 3.70 & 2.14 & 24.6 & 10.8 & 12 & 16.4 & 10.8 & 9.6 & 15.8 \\
\hline $33^{\circ}$ & 13 & $\mathrm{P} / \mathrm{IC}$ & Para perder peso & 3.68 & 2.29 & 29.8 & 9.4 & 10.5 & 11.4 & 8.8 & 12.6 & 17.5 \\
\hline $34^{\circ}$ & 38 & $\mathrm{~A} / \mathrm{F}$ & Para tener más flexibilidad & 3.58 & 2.10 & 25.4 & 9.9 & 17.3 & 12.6 & 12.3 & 8.5 & 14 \\
\hline $35^{\circ}$ & 25 & $\mathrm{~A} / \mathrm{F}$ & Para mantener la flexibilidad & 3.56 & 2.07 & 24.9 & 11.1 & 14.9 & 16.7 & 10.2 & 8.8 & 13.5 \\
\hline $36^{\circ}$ & 22 & $\mathrm{~A} / \mathrm{D} / \mathrm{B}$ & $\begin{array}{l}\text { Para disfrutar de los aspectos } \\
\text { sociales del ejercicio }\end{array}$ & 3.56 & 2.08 & 25.4 & 10.8 & 15.2 & 14 & 12 & 10.2 & 12.3 \\
\hline $37^{\circ}$ & 41 & $\mathrm{P} / \mathrm{IC}$ & Para estar más atractivo & 3.49 & 2.19 & 28.7 & 12.6 & 12.9 & 12.6 & 8.2 & 10.2 & 14.9 \\
\hline $38^{\circ}$ & 18 & $\mathrm{CE}$ & Porque ayuda a reducir la tensión & 3.44 & 2.14 & 31 & 9.6 & 12 & 13.5 & 13.2 & 7.6 & 13.2 \\
\hline $39^{\circ}$ & 46 & $\mathrm{~A} / \mathrm{D} / \mathrm{B}$ & Para hacer amigos & 3.39 & 2.19 & 33.3 & 8.8 & 13.2 & 10.8 & 11.4 & 8.8 & 13.7 \\
\hline $40^{\circ}$ & 30 & $\mathrm{C} / \mathrm{RS} / \mathrm{D}$ & $\begin{array}{l}\text { Para obtener reconocimiento } \\
\text { cuando me supero }\end{array}$ & 3.39 & 2.16 & 31.9 & 10.8 & 12 & 10.8 & 13.2 & 8.8 & 12.6 \\
\hline $41^{\circ}$ & 28 & US & Para evitar problemas cardiacos & 3.29 & 2.22 & 36.5 & 9.1 & 11.7 & 10.2 & 10.5 & 7.9 & 14 \\
\hline $42^{\circ}$ & 10 & $\mathrm{C} / \mathrm{RS} / \mathrm{D}$ & $\begin{array}{l}\text { Porque me gusta intentar ganar } \\
\text { cuando hago ejercicio }\end{array}$ & 3.25 & 2.21 & 37.4 & 8.8 & 11.4 & 10.8 & 10.5 & 7.3 & 13.7 \\
\hline $43^{\circ}$ & 42 & $\mathrm{C} / \mathrm{RS} / \mathrm{D}$ & $\begin{array}{l}\text { Para conseguir hacer cosas que } \\
\text { los demás no pueden hacer }\end{array}$ & 2.97 & 2.09 & 41.2 & 10.2 & 10.2 & 11.7 & 10.8 & 6.4 & 9.4 \\
\hline $44^{\circ}$ & 4 & $\mathrm{C} / \mathrm{RS} / \mathrm{D}$ & $\begin{array}{l}\text { Para demostrarle a los demás lo } \\
\text { que valgo }\end{array}$ & 2.83 & 1.98 & 38.9 & 14.9 & 14.9 & 8.8 & 8.2 & 6.7 & 7.6 \\
\hline $45^{\circ}$ & 36 & US & $\begin{array}{l}\text { Para recuperarme de una } \\
\text { enfermedad/lesión }\end{array}$ & 2.69 & 2.06 & 48.8 & 10.2 & 9.6 & 8.5 & 8.5 & 5.8 & 8.5 \\
\hline $46^{\circ}$ & 17 & $\mathrm{C} / \mathrm{RS} / \mathrm{D}$ & $\begin{array}{l}\text { Para comparar mis habilidades } \\
\text { con las de los demás }\end{array}$ & 2.46 & 1.92 & 51.2 & 11.1 & 13.2 & 7.6 & 5.3 & 5 & 6.7 \\
\hline $47^{\circ}$ & 23 & US & $\begin{array}{l}\text { Para evitar una enfermedad que } \\
\text { se da mucho en mi familia }\end{array}$ & 2.45 & 2.05 & 55.6 & 12 & 7 & 5 & 6.1 & 6.1 & 8.2 \\
\hline $48^{\circ}$ & 9 & US & $\begin{array}{l}\text { Porque el médico me ha } \\
\text { aconsejado hacer ejercicio }\end{array}$ & 2.38 & 1.86 & 54.1 & 10.2 & 10.8 & 8.5 & 7.3 & 3.2 & 5.8 \\
\hline . & & Bien & $\begin{array}{l}\text { C/RS/D: Competición/Recono } \\
\text { CE: Control del estrés; F/RM: }\end{array}$ & EII & UCta & & 110 & $/ 11$ & & & & \\
\hline
\end{tabular}

Finalmente, las puntuaciones más bajas en los motivos que esgrimen los adolescentes para la realización de ejercicio físico se presentan en los ítems "porque el médico me ha aconsejado hacer ejercicio" ( $\mathrm{M}=2.38$; Totalmente verdadero: 5.8\%), "para evitar una enfermedad que se da mucho en mi familia" $(\mathrm{M}=2.45$; Totalmente verdadero: $8.2 \%$ ) o "para recuperarme de una enfermedad/lesión" $\quad(\mathrm{M}=2.69 ; \quad$ Totalmente verdadero: $8.5 \%$ ), del factor urgencias de salud; y "para comparar mis habilidades con las de los demás" $(\mathrm{M}=2.46$; Totalmente verdadero: $6.7 \%)$ o "para demostrarle a los demás lo que valgo" ( $\mathrm{M}=2.83$; Totalmente verdadero: $7.6 \%$ ), del factor competición/reconocimiento social/desafio. 


\section{Discusión}

La práctica del ejercicio físico debería ser perdurable durante toda la vida del ser humano (Serra, 2008). Por lo tanto, resultará determinante conocer como poder desarrollar formas de motivación que apoyen e incentiven la realización de ejercicio físico, sobre todo en la edad adolescente, ya que se trata de un colectivo de riesgo para el abandono (Cairney, Veldhuizen, Kwan, Hay \& Faught, 2014; Samperio, Jiménez-Castuera, Lobato, Leyton \& Claver, 2016). En este contexto, el presente estudio ha analizado los factores motivacionales que inciden en el mantenimiento o la práctica del ejercicio físico según la opinión del alumnado de Educación Secundaria Obligatoria y Bachillerato, aportando información relevante para el autodiagnóstico en la adherencia a la actividad física y deportiva en la población adolescente.

Los resultados obtenidos en el análisis descriptivo confirman como factores que permiten en mayor medida incrementar la motivación hacia el ejercicio físico en adolescentes a la prevención o salud positiva y la afiliación, diversión o bienestar. Estos resultados coinciden con los señalados en los estudios realizados por Bastos et al. (2006), Marquez, Rodríguez y De Abajo (2006); Jiménez- Torres, Gordoy- Izquierdo y Gordoy (2011), Martínez et al. (2012), Moreno et al. (2012), al indicar como principales motivos a la adherencia al ejercicio físico la diversión y el mantenerse en forma.

Por el contrario, los factores motivadores con menor conexión a la práctica del ejercicio físico son las urgencias de salud y la competición, reconocimiento social o desafío. En esta línea, existen estudios que señalan que bajos niveles de competencia percibida predecía el abandono de la práctica deportiva (Guillet, Sarrazin, Carpenter, Trouilloud \& Cury, 2002), y los que indican que la competencia percibida es un fuerte predictor de la intenciones de la práctica (Taylor, Ntoumanis, Standage \& Spray, 2010).

Cabe señalar que la adherencia al ejercicio físico está condicionada por la motivación del individuo, puesto que determina el inicio, mantenimiento y abandono de una conducta. De ahí la importancia de conocer las motivaciones que impulsan a los adolescentes a la práctica o abandono del ejercicio físico, sobre todo, teniendo en cuenta los enormes beneficios que aporta a la salud y la calidad de vida. En definitiva, se debe potenciar la motivación de los adolescentes hacia el ejercicio físico, de cara a la responsabilidad con la implantación de estilos de vida activos y perennes a lo largo del tiempo.

\section{Referencias bibliográficas}

Bastos, F.C., Barhum, R.A, Alves, M.V., Bastos, E.T., Mattar, M.F., Rezende, M.F., Mardegan, M., \& Bellangero, G. (2006). Perfil do administrador esportivo de clubes sócio-culturais e esportivos de São Paulo/Brasil. Revista Mackenzie de Educação Física e Esporte, 1(5), 13-22.

Cairney, J., Veldhuizen, S., Kwan, M., Hay, J., \& Faught, B. E. (2014). Biological Age and Sex-Related Declines in Physical Activity during Adolescence. Medicine and Science in Sports and Exercise, 46(4), 730-735.

Capdevila, Ll., Niñerola, J., \& Pintanel, M. (2004). Motivación y actividad física: el autoinforme de motivos para la práctica de ejercicio físico (AMPEF). Revista de Psicología del Deporte, 13(1), 55-74.

Cecchini, J. A. (2011). Achievement goals, social goals, and motivational regulations in physical education settings. Psicothema, 23(1), 51-57.

Guillet, E., Sarrazin, P., Carpenter, P., Troullioud, D., \& Cury, F. (2002). Predicting persistence or withdrawal in female handballers with Social Exchange Theory. International Journal of Sport Psychology, 37, 92-104.

Jaakkola, T., Washington, T., \& Yli-Piipari, S. (2013). The association between motivation in school physical education and self-reported physical activity during Finnish junior high school: A self-determination theory approach. European Physical Education Review, 19(1), 127-141

Jiménez- Torres, M.G., Gordoy- Izquierdo, D., \& Gordoy, J.F. (2011). Relación entre los motivos para la práctica físico-deportiva y las experiencias de flujo en jóvenes: diferencias en función del sexo.Universitas Psychologica, 11(3), 902-920.

Kulavic, K., Hultquist, Ch.N., \& McLester, J.R. (2013). A Comparison of Motivational Factors and Barriers to Physical Activity Among Traditional Versus Nontraditional College Students. Journal of American College Health, 61, 60-66.

Markland, D., \& Ingledew, D.K. (1997). The measurament of exercise motives: Factorial validityand invariance across gender of a revised Exercise Motivations Inventory. British Journal of Health Psychology, 2, 361-376.

Márquez, S., Rodríguez, J., \& De Abajo, S. (2006). Sedentarismo y salud: efectos beneficiosos de la actividad física. Apunts Educación Física y Deportes, 83, 12-24.

Martínez, A.C., Chillón, P., Martín-Matillas, M., Pérez, I., Castillo, R., Zapatera, B., ...\& Delgado-Fernández, M. (2012). Actitudes hacia la práctica de actividad físico-deportiva orientada a la salud en adolescentes españoles: Estudio AVENA. Retos. Nuevas tendencias en Educación Física, Deporte y Recreación, 22, 43-48.

Moreno, J.A., Martínez, C., Moreno, V., Marcos, P., Conte, \& L., Moreno, R. (2012). Motivación, creencias de habilidades e intención de ser físicamente activo al finalizar la Educación obligatoria. Revista Mexicana de Psicología, 29(2), 175-183.

Peeters, M., Wiers, R., Monshouwer, K., Van de Schoot, R.A.J.G., Janssen, T., \& Vollebergh, W. (2012). Automatic processes in at-risk adolescents: the role of alcohol-approach tendencies and response inhibition in drinking behavior. Addiction, 107, 1339-1346.

Samperio, J., Jiménez-Castuera, R., Lobato, S., Leyton, M., \& Claver, F. (2016). Variables motivacionales predictoras de las barreras para la práctica de ejercicio físico en adolescentes. Cuadernos de Psicología del Deporte, 16(2), 65-76.

Serra, J.R. (2008). La actividad física en la población adolescente de la provincia de Huesca. Tesis de doctorado, Universidad de Zaragoza. Recuperado de 
http://zaguan.unizar.es/record/1903/files/TUZ_0027_s erra_factor.pdf

Taylor, I., Ntoumanis, N., Standage, M., \& Spray, C. (2010). Motivational predictors of physical education students' effort, exercise intentions, and leisure-time physical activity: A multilevel linear growth analysis. Journal of Sport and Exercise Psychology, 32, 99-120.

Vergara, P., Trevisi, N., Ricco, A., Petracca, F., Baratto, R., Cireddu, M. Bisceglia, C., Maccabelli, G., \& Della, P. (2012). Late Potentials Abolition as an Additional Technique for Reduction of Arrhythmia Recurrence in Scar Related Ventricular Tachycardia Ablation. Journal of Cardiovascular Electrophysiology, 23(6), 621-627. doi: 10.1111/j.1540-8167.2011.02246.x 